\title{
Gadamer, O'Gorman, Levi. Combates contra el neopositivismo historiográfico y contra el fetiche del documento
}

Morelos Torres Aguilar Universidad de Guanajuato

Resumen

El artículo analiza críticamente, a partir de las consideraciones de Giovanni Levi, Hans-Georg Gadamer y Edmundo O'Gorman, la manera en que los historiadores aprehenden los acontecimientos y el procedimiento mediante el cual obtienen y utilizan sus fuentes. Las ideas de estos tres pensadores cuestionan, desde distintas perspectivas, la preeminencia de una heurística basada sólo en la afanosa búsqueda de la verdad, característica de la escuela científica alemana y el positivismo, corrientes ambas del siglo XIX; proponen en cambio, como alternativa que mucho debe a Ser y tiempo de Heidegger, la comprensión e interpretación de los procesos y los sujetos históricos, como una forma adecuada de explicación histórica.

Palabras clave: Historiografía, neopositivismo, documento, verdad, comprensión. 
Abstract

The article critically analyzes, from the considerations of Giovanni Levi, Hans-Georg Gadamer and Edmundo O'Gorman, the way in which the historians apprehend the events and the procedure by means of which they obtain and use his sources. The ideas of these three thinkers question, from different perspectives, the preeminence of a heuristic based only on the eager search of the truth, characteristic of the scientific german school and the positivism, currents both of the $19^{\text {th }}$ century; they propose on the other hand, as alternative that much owes to Being and time of Heidegger, the comprehension and interpretation of the processes and the historical subjects, as a suitable form of historical explanation.

Keywords: Historiography, Neopositivism, Document, Truth, Comprehension.

\section{Giovanni Levi: el documento, fragmento de una realidad incierta}

$\mathrm{Z}^{\mathrm{n}}$ julio de 2011, en el marco de un congreso internacional Csobre historia de la educación, Giovanni Levi pronunció una ponencia magistral. ${ }^{1}$ El contexto de la presentación no podía ser mejor: el auditorio estaba atestado, los asistentes lucían expectantes y la merecida fama del historiador italiano había contribuido a generar interés, incluso emoción, en todos aquellos que colmaban el recinto.

Levi no se conformó con describir o fundamentar las conocidas propuestas de la microhistoria italiana, sino que lanzó dos

\footnotetext{
${ }^{1}$ Se trataba de la 33 International Standing Conference for the History of Education (ISCHE), celebrada en San Luis Potosí. (Todas las referencias a Levi, de aquí en adelante, corresponden a esta conferencia).
} 
ideas tan contundentes como provocadoras. La primera comenzaba con una pregunta: "¿por qué los historiadores, cuando elaboran los marcos téoricos de sus proyectos de investigación, no asumen como parte de su argumentación las ideas de otros historiadores, sino - por lo general — las propuestas teóricas de científicos sociales tales como los sociólogos, los antropólogos y los politólogos?” La respuesta que dio el historiador italiano guardaba, dentro de su sencillez, una sutil reclamación dirigida a los propios historiadores: "Los consultamos — decía— porque ellos sí hicieron la tarea. Ellos sí reflexionan sobre la sociedad, sobre el ser humano, sobre la propia historia, mientras que los propios historiadores se dedican a trabajar laboriosamente, esforzadamente, en sus pequeñas parcelas. Aquellos son quienes proponen vastas interpretaciones capaces de unir en una explicación inteligente los pequeños fragmentos que producen los historiadores".

La segunda idea de Levi, aún más perturbadora, fue planteada en la parte final de la conferencia, en la sesión de preguntas, cuando una colega le preguntó: “¿Qué experiencia importante nos puede transmitir a partir de su trayectoria como investigador?". La respuesta del historiador italiano fue contundente: "Que la historia no se hace con documentos" y, aún, añadió: "Sólo los historiadores jóvenes, los que comienzan a realizar sus investigaciones, consideran que la historia se realiza con documentos".

$\mathrm{Si}$ la frase de Levi fue sorprendente, no lo fue menos la reacción del público. El auditorio enmudeció y los historiadores presentes evidenciaron de diversas maneras su nerviosismo y desconcierto. El pasmo de la concurrencia puede ser explicado fácilmente si consideramos que el quehacer de la mayoría de los historiadores se basa precisamente en el documento, con toda la parafernalia que ello implica: la búsqueda de la fuente primaria, del escrito inédito, de la carpeta escondida en un rincón secreto del archivo; la discreta pero cotidiana competencia para ver quién cita más documentos 
en sus obras, quién los ha hallado más antiguos, quién ha viajado más para encontrarlos, quién ha sabido hablar más bonito en el oído de archiveros, funcionarios, bibliotecarios, familiares del sabio personaje fallecido, para localizar el fundamental, el único, el auténtico documento sin el cual sería imposible escribir la historia de determinada colectividad.

Sobre la primera idea de Levi aquí referida, que describe el esforzado trabajo del historiador en su pequeña parcela, Carlo Ginzburg, también perteneciente a la corriente denominada microhistoria italiana, cita un texto escrito por el historiógrafo holandés F. R. Ankersmit, donde se revela la tendencia - típica en la historiografía posmoderna- de "concentrar la atención sobre los fragmentos antes que sobre conjuntos más vastos"; para explicarla con sencillez, se sirve de una metáfora vegetal:

En el pasado, los historiadores se ocupaban del tronco del árbol o de las ramas; sus sucesores posmodernos se ocupan únicamente de las hojas, o sea de fragmentos minúsculos del pasado que investigan de forma aislada, independientemente del contexto más o menos amplio (las ramas, el tronco) del que formaban parte (Ankersmit, 1989: 143).

A juicio de Ginzburg, Ankersmit defiende esta postura posmoderna porque considera que "la ambición de conocer el pasado ha decaído: el significado de los fragmentos se busca en el presente, en la forma 'en que su configuración puede ser adaptada a formas de civilización existentes en la actualidad”. En todo caso, se trata de una renuncia a dotar a la historiografía de cualquier valor cognoscitivo y, al mismo tiempo, de una apuesta por el valor narrativo del texto histórico.

El abandono de la explicación vasta, de la comprensión totalizadora en el trabajo del historiador, suele articularse a cierta metodología reduccionista, la cual deliberadamente se aleja del método 
comparativo, por ejemplo. De esta manera, la explicación sobre la realidad observada se agota en sí misma y, por ello, se evita estudiar la relación que guarda con otras realidades, como si los objetos de estudio constituyeran entes aislados, desligados de las circunstancias vitales existentes en otras localidades, en otros países e incluso en otros continentes. Pero si bien en la narrativa el relato de pequeńas colectividades, incluso de ciertos personajes, resulta muy importante por la empatía o identificación que éstos despiertan en el lector, existe también un público que desea encontrar en la historia claves más extensas, que le permitan conocer un significado amplio de la humanidad en su conjunto. Es ahí donde comienza el trabajo del antropólogo, del economista, del sociólogo, que organizan los fragmentos generados por los historiadores, a fin de ofrecer una explicación multiabarcante del ser humano. Es el caso, por ejemplo, del sistema mundo de Wallerstein, que tantos seguidores ha tenido en la actualidad, precisamente porque ofrece, tanto a especialistas como a legos, una visión totalizadora de la sociedad, de su pasado e incluso de su futuro.

Sin embargo, es aún más interesante la segunda idea planteada por Levi en su conferencia: la perspectiva crítica en torno a la idea que se tiene tradicionalmente sobre el documento. En principio, sabemos que lo dicho en aquella ocasión por el historiador italiano era sólo una reafirmación de sus ideas en torno al aprovechamiento ceremonioso y reverencial, casi místico, que muchos historiadores hacen del propio documento. En una entrevista realizada por Santiago Muñoz Arbeláez y María Cristina Pérez Pérez, Levi explica de manera clara y sumamente sencilla las notables limitaciones que tienen los documentos y, por tanto, las obras historiográficas que se basan ciegamente en ellos:

El tema que considero central de la biografía es la incertidumbre, y los historiadores la han abolido completamente de su actividad. En lugar de buscar complejidad, los historiadores asumen 
que los individuos enfrentan el mundo de manera racional. Pero el panorama es distinto... nosotros dedicamos el 95\% de nuestra actividad a la inactividad, la reflexión, las elecciones continuas, la incertidumbre y los temores. Sin duda, tales aspectos son fundamentales en la vida y los historiadores tienen dificultades, porque nosotros trabajamos sobre restos o evidencias muy fragmentarias de la vida de las personas: los documentos. Los documentos se crean sólo de acciones o cosas que ocurrieron. No hay un documento de la indecisión, la duda o la incertidumbre. Es por eso que debemos trabajar también con los no documentos, dándole sentido a estas huellas en su contexto (Muñoz, 2010: 202).

De ahí la importancia que cobran otras fuentes, como las entrevistas de la historia oral, para explicar ese vastísimo territorio que ocupan aquellas actividades, actitudes, convicciones, sentimientos e ideas que, precisamente por no haber ocurrido, por no haberse constituido en acciones, sino posiblemente sólo en pensamientos o en actos aparentemente irrelevantes para el acontecer de los grandes núcleos de población, no han tenido cabida en los documentos históricos y, por tanto, no han sido recogidos por los historiadores para la construcción de sus escritos.

Desde otra perspectiva, aunque también a partir de la vertiente reflexiva de la microhistoria italiana, Carlo Ginzburg recuerda el ensayo "Partenza di un gruppo di soldati per la Libia", escrito en 1912, donde Renato Serra reflexiona sobre la relación entre realidad y documento:

Hay alguna gente que de buena fe se imagina que un documento puede ser una expresión de la realidad... Como si un documento pudiese expresar algo distinto de sí mismo... Un documento es un hecho. La batalla es otro hecho (infinidad de otros hechos). Los dos no pueden ser uno... El hombre que trabaja es un hecho. Y el hombre que narra es otro hecho... Todo testimonio responde 
solamente de sí mismo; del momento propio, del propio origen, del propio fin, y de nada más... (Ginzburg, 1994: 36).

Con base en la observación de Serra, se colige la dificultad de fundar la verdad en los propios documentos, de pensar que en ellos está contenida la verdad que afanosamente buscan los historiadores. Dado que el documento, como hecho en sí mismo, puede no revelar algo más que su propio, su particular significado.

Miguel Ángel Beltrán observa que, ya desde la postura de los primeros Annales, Lucien Febvre y Marc Bloch advertían que el hecho histórico no existe en forma pura en los documentos, pues "siempre hay una refracción al pasar por la mente de quien los recoge". Más adelante, observa que a partir de la obra de Michel de Certeau:

se replantea la noción misma de documento considerado, cada vez menos, la prueba de la verdad, el rastro que permite la reconstrucción del pasado, para dar lugar a un trabajo más desde su interior: la historia organiza, recorta, distribuye y ordena; distingue lo que es pertinente y lo que no lo es; trata de definir conjuntos, series, relaciones... se diría así, siguiendo la propuesta de Chartier, que los documentos históricos, al igual que los libros de lectura, están revestidos de significaciones plurales y cambiantes en el punto de articulación entre la proposición y su recepción, entre las formas y motivaciones que originan su estructura discursiva y las capacidades y expectativas de los públicos que se adueñan de él (Beltrán, 2001: 33).

Así que un ente cuya veracidad parecería a muchos tan obvia, como lo es el documento, se halla en realidad revestido de un horizonte de significación muy amplio, que es asimilado en las narrativas historiográficas de un modo completamente distinto, dependiendo de la lectura que hace cada autor. El mismo documento puede dar pie a valoraciones e interpretaciones divergentes a partir de la 
recepción de los lectores. Esto aún sin considerar que muchas veces los documentos contienen datos internos que resultan contradictorios entre sí.

En este mismo sentido, Ronen Man recuerda que "la microhistoria rompe con la manera tradicional y positivista en que se entienden los documentos en tanto fuentes privilegiadas de la historia”, pues afirma que

según Giovanni Levi, los documentos son, por sus características, falsos y parciales. Sólo pueden darnos ciertos indicios que sirven a la manera de sugerencias. Los mejores documentos serán los que no entendemos, los que no son evidentes ni inmediatos y que nos pondrían en problemas y contradicciones. Esto evidentemente introduce un quiebre con la manera tradicional de pensar a los documentos como "espejos transparentes" y objetivos de una realidad en tanto hechos sociales aprehensibles racionalmente de manera inmediata. Para la microhistoria los documentos son sólo fragmentos huidizos de lo real, por lo tanto deben "leerse" oblicuamente y de forma indirecta, a la manera del análisis del discurso freudiano (Man, 2013: 169).

De acuerdo con Man, entonces, el documento no es un ente que denota, sino más bien uno que connota, un ente polisémico que no representa la verdad en sí mismo, sino una aproximación a ésta. De ahí el carácter de mediador que posee el documento, o como lo dirá O’Gorman más adelante: de intermediario, de vía de acceso a una realidad que, aunque separada del observador por el tiempo, actúa sobre éste y le plantea numerosas interrogantes.

Ahora bien, aunque sabemos que el origen del culto que el historiador rinde al documento se halla en el siglo XIX, una buena manera de entenderlo consiste en compararlo con las épocas que le antecedieron. Para hacer esto, Beatriz Bragoni parte de la idea de Carlo Ginzburg de que "la tradición clásica le exigía a la exposi- 
ción histórica la capacidad de representar con vivacidad personajes y situaciones". Desde esta perspectiva el historiador, al igual que lo hace un abogado, tenía que "convencer por medio de un argumento eficaz y comunicar la ilusión de la realidad, no sólo por medio de la producción de pruebas o de la valoración de pruebas producidas por otros". Sin embargo — recuerda la historiadora argentina- la práctica y la perspectiva de los historiadores se vio modificada a partir del siglo XVIII; ya en el siglo XIX, la historiografía generada por la escuela científica alemana se basaba claramente en documentos, al erigirse en algo así como un tribunal reconocido. "A partir de entonces [plantea Bragoni], el dilema entre juzgar o comprender atravesó el campo disciplinar" (1998: 142).

Aquel viejo dilema, no resuelto, sigue explicando por qué el historiador escribe su historia. Para unos, los partidarios del juicio, es decir de la búsqueda de la verdad, lo más importante es conocer lo que verdaderamente sucedió, según la proposición de Ranke. Por eso buscan afanosamente dicha verdad en los documentos que consultan. De ahí la importancia que guardan éstos en su explicación del pasado. Otros, los partidarios de la comprensión, siguen la idea de que la verdad es más bien una aspiración, en lugar de un fundamento incontrovertible de la explicación histórica.

\section{Hans-Georg Gadamer: verdad o comprensión}

En algunos parágrafos de Verdad y método, Hans-Georg Gadamer señala el problema de la verdad. Por ejemplo, cuando se refiere a ésta como verificabilidad, ponderando su carácter ideal o idealizado, cuando no francamente infecundo:

Todos hemos de aspirar como ideal a la verificabilidad de todos los conocimientos dentro de lo posible. Pero hemos de reconocer que este ideal se alcanza pocas veces, y que los investigadores que 
aspiran a alcanzar este ideal con la mayor precisión, no suelen decirnos las cosas realmente importantes (Gadamer, 1998: 55).

Por este motivo, Gadamer observa: "hemos de reconocer que los resultados más importantes y fecundos alcanzados en las ciencias del espíritu quedan muy al margen del ideal de verificabilidad" (1998: 57).

Porque para Gadamer, lo que constituye al investigador como tal es "la capacidad de apertura para ver nuevas preguntas y posibilitar nuevas respuestas... El que intenta comprender un texto está dispuesto a dejar que el texto le diga algo" (1998: 66). De este modo, Gadamer toma partida por la comprensión frente a la pretensión de verdad de una tradición historiográfica proveniente de la Ilustración, tradición fundada precisamente por aquellos que, asombrados por el avance de la ciencia, en particular de la Física, reclamaban para el conocimiento histórico un rigor similar y una capacidad de demostración que, en realidad, no poseen las humanidades, a las que Gadamer llama las ciencias del espíritu. Por ello, de éstas, Gadamer extrae no la certidumbre pretendidamente proporcionada por el método, sino más bien la profundidad que se adquiere, precisamente, cuando se ha trascendido el propio método:

En las ciencias del espíritu abrigamos una fundada desconfianza hacia un determinado tipo de trabajos científicos que muestran demasiado a las claras... el método con el que están hechos. ¿Buscan esos trabajos algo nuevo? ¿Llegan realmente al conocimiento de algo? ¿O imitan tan perfectamente el método de conocimiento y sus formas externas, que producen la impresión de un trabajo científico? (Gadamer, 1998: 57).

Precisamente basado en esta perspectiva trascendente que busca realmente el conocimiento de algo, el filósofo alemán propone a 
la hermenéutica como vía de acceso a la realidad histórica, pues para ella "comprender e interpretar textos no es sólo una instancia científica, sino que pertenece con toda evidencia a la experiencia humana del mundo" (Gadamer, 1999: 23).

De este modo, Gadamer, al introducir en el ejercicio del historiador — es decir de quien comprende los hechos, del intérpreteel concepto de experiencia, abre un universo de significados para los actos realizados por los actores de la historia. Porque de acuerdo con la experiencia - aquí podemos recuperar la idea de incertidumbre de Levi, expresada anteriormente-, la mayoría de las acciones de los seres humanos no son racionales, ni producto de un propósito claro; muchas obedecen a factores como la intuición, la desconfianza, la emoción, o bien son contradictorias con respecto a otras realizadas por los propios individuos. En este sentido, Gadamer permite entender que, junto con la certidumbre, existe en la explicación histórica un principio de duda, de incertidumbre, de atención, de diálogo, en donde las ideas no sólo se afirman, sino que de la misma manera se discuten, se debaten, se comparten: "Cuando se oye a alguien o cuando se emprende una lectura, no es que haya que olvidar todas las opiniones previas sobre su contenido, o todas las posiciones propias. Lo que se exige es simplemente estar abierto a la opinión del otro o a la del texto" (Gadamer, 1999: 335).

Esto implica, por supuesto, la necesidad de aceptar como fuentes una multiplicidad de voces, multiplicidad de documentos, de fuentes discriminadas por la historiografía tradicional, siempre y cuando éstas tengan algo qué decirnos, siempre que aporten elementos para la comprensión de la realidad histórica, pues ésta es precisamente la realidad humana.

Por eso, la relación entre el documento y el acontecimiento no constituye una cándida línea, simple y llana, perfectamente trazada y entendida (como lo proponía la historiografía científica alemana del siglo XIX), sino un círculo o conjunto de círculos, una espiral 
y, tal vez, hasta un laberinto que sólo en parte se logra develar, según lo escribe Gadamer: "El movimiento de la comprensión va constantemente del todo a la parte y de ésta al todo. La tarea es ampliar la unidad del sentido comprendido en círculos concéntricos" (1999: 361).

\section{Edmundo O'Gorman:}

la inexistencia del documento indispensable

En su obra Crisis y porvenir de la ciencia histórica, publicada en 1947 y reeditada recientemente, ${ }^{2}$ Edmundo O'Gorman enuncia algunos aspectos de su idea de la historia y aporta una forma renovadora de escribir textos históricos que abandona la tradición de la historiografía científica alemana y del positivismo, en aras de la comprensión histórica. Por supuesto, su propuesta constituye una visión absolutamente provocadora, que en su momento escandalizó a no pocos historiadores, pero que al mismo tiempo proporcionó a éstos una perspectiva lo suficientemente crítica como para que entendieran que la teoría, la ciencia y el oficio del historiador necesitaban urgentemente de una renovación no sólo de las formas, sino de los principios. En esta tesitura, la obra parte de la forma en que es vista la historia en su época, nada menos que como un cadáver:

He allí el cadáver, el cuerpo visible de la historia...: gran total de todas esas cosas fichadas y por fichar, catalogadas, hacinadas, amontonadas, bien medidas y mejor pesadas, que son las llamadas "fuentes"... el historiador científico... por lo general no es parco en cumplir, como una tarea de simple relator de esos materiales... (O’Gorman, 2006: 76).

${ }^{2}$ La edición de la UNAM de 2006 hace justicia a un texto que había sido injustamente olvidado o postergado por medio siglo. 
Es decir, el historiador de los años cuarenta del siglo pasado se conformaba con aquello que hasta la fecha es llamado acopio de las fuentes: una operación mecánica, cuya erudición se reducía a seleccionar adecuadamente las obras indiscutibles, los documentos novedosos, para luego, a partir de ellos, escribir nuevas obras en donde, negando cualquier responsabilidad propia y cobijado por la bandera de la veracidad, se limitaba a transcribir, a repetir, a decir que esto o aquello ocurrió, porque las fuentes así lo mencionan. Contra esta operación mecánica de selección y ponderación de fuentes se lanza O'Gorman. Para él las fuentes novedosas, inéditas, hasta ahora desconocidas, de primera mano, etc., no son más relevantes que otras fuentes cualesquiera:

Para la verdadera ciencia de la historia, no hay "materiales" y "fuentes" en el sentido que usualmente se concede a esos términos. Sólo hay intermediarios... por eso la llamada "selección de fuentes", a que concede tanta importancia la historiografía, carece de sentido... como lo que verdaderamente interesa al historiador es entrar y mantener el contacto con la realidad de la historia, nada importa cuáles sean los medios por donde se realiza ese contacto... ninguna de las llamadas "fuentes" pueden ser de suyo ni más ni menos verdaderas que otras, por la decisiva razón de que la verdad no es algo contenido en ellas. Todo lo que puede concederse a favor de las "fuentes de primera mano" es que son intermediarios más primitivos, lo que no quiere decir que sean ni más ni menos dignos de fe que los otros... por parejos motivos ha de afirmarse que carecen de fundamento verdadero aquellas exigencias que obligan al historiógrafo, so pena de caer en completo desprestigio, a emplear en cada nueva producción suya "materiales inéditos", "nunca antes vistos", o "hasta ahora desconocidos", así como a demostrar a satisfacción de los "círculos eruditos" de que está "enterado" de todo cuanto hasta ese momento se ha escrito o dicho acerca del tema que lo ocupa... (O'Gorman, 2006: 260-262). 
O'Gorman apenas calienta motores en su vehículo impulsado por la crítica. En otra parte de su obra, se enfrenta a las famosas autoridades, a los autores incontrovertibles, a las obras indudables, a los hechos aparentemente demostrados con tal cantidad de pruebas, que no cabría duda alguna respecto a lo que aquellos sabios inefables han escrito: "No hay 'autoridades' —escribe-: sólo hay vías de acceso" (O’Gorman, 2006: 263).

Todavía más. El historiador ataca los cimientos y la tradición del oficio del historiador, pues ésta suele basarse en la recopilación de numerosos documentos y centenares de fuentes, a partir de la creencia de que el número de pruebas podrá dar una versión más sólida e incuestionable sobre lo sucedido. Asimismo, dicha tradición contiene un criterio básico con el que son aceptadas o discriminadas las fuentes: el de la singularidad, la originalidad o incluso el carácter inédito o desconocido de la fuente en cuestión. Escribe don Edmundo, en forma implacable:

Un testimonio vale tanto como mil, y es absolutamente indiferente que se trate de documentos impresos o inéditos, raros o habituales, recordados u olvidados, recientes o antiguos. ¿¿Cuántas y cuales "fuentes" deben emplearse en tal caso? Se puede contestar que el verdadero historiador emplea las que le vengan en gana; que no importa si son una o cien, ni menos si son inéditas o no, pues que sólo son hilos conductores... para la comprensión auténtica, todo es intermediario, todo es "fuente de primera mano" (2006: 264).

¿Podría culpar alguien a nuestro historiador por ir aún más allá, más lejos? Porque, con un eco de la obra de Protágoras, reúne estrechamente la libertad del investigador con su responsabilidad y resalta el albedrío que necesariamente tiene que asumir quien escribe la historia para dar su propia versión de los hechos, más 
allá de los pocos o numerosos documentos que hubiere podido consultar:

Es el hombre quien funda la verdad de las cosas, y por eso es insensato coartar con exigencias metódicas su libertad para elegir los intermediarios de que se vale en el descubrimiento de la realidad... Y aunque es cierto que de ordinario es más fácil partir de intermediarios más originales, no se piense que son insustituibles. La posibilidad de descubrir la realidad radica en nuestra existencia, no en las cosas (O'Gorman, 2006: 264).

O’Gorman termina su apasionada crítica contra los historiadores tradicionales proponiendo una metáfora extrema, cuasi apocalíptica, sobre la posibilidad o imposibilidad del conocimiento histórico: "si por obra de un mago compasivo y poderoso desaparecieran todos los ficheros, todos los catálogos y todas las bibliotecas y archivos del mundo, las posibilidades de fundar la verdad histórica quedarían intactas en medio del lloriqueo universal de los eruditos" (O’Gorman, 2006: 265).

\section{Conclusiones}

Resulta claro que en este tiempo nuestro, en la segunda década del siglo XXI, estamos presenciando un resurgimiento del culto al documento, donde éste es considerado como una suerte de fetiche, una piedra filosofal a partir de la cual es posible convertir a los acontecimientos en verdades indudables y al relato en respetable historia. Se trata de una especie de neopositivismo, para el cual sólo la prueba del documento puede dotar de verdad a un relato. Debido a ello la labor del historiador debe limitarse exclusivamente a encontrar las fuentes adecuadas para explicar el fenómeno, y a transcribir con fidelidad lo que éstas dicen. A partir de dicho propósito, el investigador realiza el procedimiento llamado crítica 
de fuentes, mediante el cual determina la veracidad o falsedad de los datos que aparecen en el documento. Cabe señalar que, con base en esta misma crítica, son rechazadas por lo general fuentes tales como entrevistas de historia oral o notas periodísticas, debido a que es necesario "tomarlas con pinzas", es decir, separar en ellas la verdad de la falsedad, la realidad de la imaginación, etc.

Con el ánimo de atender a dicha tendencia, los historiadores suelen fundar sus trabajos en dos premisas irreductibles: $a$ ) la pretensión de que por medio de sus trabajos es posible conocer verdaderamente lo que sucedió; $b$ ) la satisfacción de haber elaborado sus obras mediante la utilización de fuentes de alta calidad, documentos inéditos o poco conocidos, libros que nunca más se han vuelto a publicar, cartas privadas y virginales, afanosamente buscadas en archivos secretos o bien casualmente descubiertas entre muchos otros manuscritos. Sin embargo, a la luz de las propuestas teóricas de Giovanni Levi, de Hans-Georg Gadamer y de Edmundo O'Gorman, tales premisas resultan no sólo erradas, sino absolutamente innecesarias. Porque si reconocemos que durante más de dos siglos no hemos podido librarnos de la penosa disyuntiva entre conocer la verdad o comprender los sucesos, podemos coincidir en que es más valioso y fecundo el segundo camino, pues la verdad - la verificabilidad, como lo escribe Gadamer - no deja de ser, finalmente, más que una poderosa, ilusoria y reputada aspiración; mientras que la comprensión constituye un proceso dinámico, abierto a distintos datos e interpretaciones y, sobre todo, de carácter plenamente histórico, puesto que cada generación genera sus propias evidencias, su manera propia de comprender e interpretar los mismos acontecimientos, o bien, se enfoca a unos personajes, unas circunstancias y unas acciones distintas a aquellas que fueron enfatizadas por generaciones anteriores.

Una interesante prueba de lo anterior la constituyen las no tan recientes conmemoraciones por el centenario y el bicentenario de 
la Revolución Mexicana y del inicio de la Revolución de Independencia en México, respectivamente, que tuvieron lugar en 2010. En aquella ocasión diversos historiadores propusieron nuevas lecturas sobre dos acontecimientos, que ya han sido de por sí estudiados minuciosamente, o bien nuevos temas relacionados con éstos. Por supuesto, sus nuevas interpretaciones no dependieron sólo del hallazgo de nuevos documentos, sino de la experiencia vital de los propios investigadores e incluso de su pertenencia a determinadas generaciones, las cuales habían disfrutado o padecido, en forma conjunta, ciertos acontecimientos que las distinguieron, precisamente, como tales.

En vista de todo lo que aquí se ha escrito, parece adecuado terminar con aquella frase pronunciada por nuestro sabio Alfonso Caso en las inolvidables sesiones organizadas en 1945 por la Sociedad Mexicana de Historia: "la verdad histórica es sólo probabilidad" (Matute, 1974: 47).

\section{Bibliografía}

Ankersmit, F. R., 1989, "Historiography and Posmodernism", en History and Theory, vol. 28, núm. 2 (mayo), Middletown, CT, Wesleyan University, pp. 149-150.

Beltrán, Miguel Ángel, 2001, "Pensar la historia en ¿̇tiempos posmodernos?", en Memoria y civilización $(M y C)$, núm. 4, Pamplona, Universidad de Navarra, pp. 19-41.

Bragoni, Beatriz, 1998, "Historiografía, microhistoria. Algunas consideraciones adicionales en torno a un tema recurrente", en CUYO. Anuario de Filosofía Argentina y Americana, vol. 15, Mendoza, Universidad Nacional de Cuyo, pp. 135-148.

Gadamer, Hans-Georg, 1999, Verdad y método I, Salamanca, Sígueme. 
1998, Verdad y método II, Salamanca, Sígueme.

Ginzburg, Carlo, 1994, "Microhistoria: dos o tres cosas que sé de ella”, Manuscrits: revista d'història moderna, núm. 12 (enero), pp. 13-42.

Man, Ronen, 2013, "La microhistoria como referente teórico-metodológico. Un recorrido por sus vertientes y debates conceptuales", Historia Actual Online, núm. 30 (Invierno), pp. 167173.

Matute, Álvaro, 1974, La teoría de la historia en México (19401973), México, SEP.

Muñoz Arbeláez, Santiago y María Cristina Pérez Pérez, 2010, "Perspectivas historiográficas: entrevista con el profesor Giovanni Levi”, Historia Crítica, núm. 40 (enero-abril), pp. 197205.

O'Gorman, Edmundo, 2006, Crisis y porvenir de la ciencia histórica, México, UNAM / Coordinación de Humanidades.

(Artículo recibido el 7 de enero de 2014; aceptado el 25 de febrero de 2014) 